\title{
NÍVEL DE ANSIEDADE PRÉ-COMPETITIVA EM ATLETAS DE NATAÇÃO
}

Marcella Manfrin Barbacena

Roseni Nunes de Figueiredo Grisi

\section{Resumo}

O estudo visou avaliar os níveis de ansiedade pré-competitiva de atletas de natação em duas competições de diferentes níveis: estadual (CP) e regional (CR). Participaram do estudo 37 atletas, sendo 21 do sexo feminino, de três clubes do estado da Paraíba. Eles responderam ao questionário de ansiedade précompetitiva (CSAI-2) 15 minutos antes de suas provas. O teste revelou níveis de ansiedade précompetitiva maiores em competições mais importantes (CR), com 36,8\% dos sujeitos apresentando ansiedade média e alta em CR e apenas $11,5 \%$ na CP. Além disso, as mulheres apresentaram uma tendência para serem mais ansiogênicas que os homens. Todos os atletas apresentaram um bom nível de autoconfiança independentemente do nível da competição, mas com tendência para aumento de acordo com o nível da competição. Este trabalho contribui para uma reflexão da ansiedade em situações competitivas, possibilitando assim, conhecer como variam os estados de ansiedade e de autoconfiança antes de uma competição, sabendo que a ansiedade poderá afetar positivamente ou negativamente no rendimento posterior dos atletas, devendo ser motivo de atenção aos técnicos, psicólogos e estudiosos da área.

\section{Palavras-Chave:}

Ansiedade; Natação; Gênero.

\section{LEVEL OF ANXIETY PRE-COMPETITIVE IN ATHLETES IN SWIMMING}

Marcella Manfrin Barbacena

Roseni Nunes de Figueiredo Grisi

\begin{abstract}
This study aimed to investigate the pre-competitive anxiety levels of swimming athletes in two distinct competitive levels: State (CP) and Region (CR). Participated to study 37 athletes, 21 femme in to three differents clubs to Paraíba State. Their answered the i pre-competitive anxiety 15 min before their swimming test. The inventory revealed significant higher pre-competitive levels in the more important competitions (CR), with 36,8\% the athletes to present anxiety like medium and higher, and just $11,5 \%$ in CP. After this, the femme presented a inclination for more anxiety than men. For other side, all athletics presented a good level for self-esteem independent the competitive level, but with tendency to raise in accordance with the level of the competition. This work contributes for a reflection on the action of the anxiety in competitive situations, making possible to know how varies the states of anxiety and selfconfidence before a competition, knowing that this will be able to affect positive or negative the results of the athletes, having to be attention for the choach, psychologists and of the area.
\end{abstract}

\section{Key-Words:}

Anxiety; Swimming; Genders. 


\section{Introdução}

Sendo um contexto natural com situações de elevada exigência e competitividade, o desporto de rendimento é usado pelos psicólogos para a compreensão do comportamento humano, das emoções e dos processos de adaptação humana (HACKFORT; SPIELBERGER, 1989). O sucesso, as críticas sofridas e a oportunidade tão esperada pelo atleta do alto rendimento, são algumas das suas tensões vivenciadas e, desta forma, pode ser alguns fatores ansiogênicos, pois dependem da percepção e da interpretação que cada pessoa tem dos acontecimentos (DAMÁZIO, 1997).

Um dos campos mais abordados pela Psicologia do Desporto é a ansiedade e as suas relações com o rendimento. A experiência de stress e ansiedade na competição desportiva constitui um problema usual e preocupante para todos aqueles que, direta ou indiretamente, se encontram envolvidos no desporto (CRUZ, 1996b; SCANLAN, 1984).

De acordo com as pesquisas de Parfitt apud Gonçalves (1998) a ansiedade não tem o mesmo efeito em todas as pessoas e nem em todos os esportes. A ansiedade somática está mais ligada a esportes de exigências aeróbicas (lutas, corridas, natação, etc), enquanto a ansiedade cognitiva estará mais ligada a esportes competitivos que envolvem estratégias (xadrez, golf, sinuca, etc). Nos desportos individuais, como a natação, a iniciativa, determinação, persistência, coragem e motivação são determinantes nos resultados, fazendo com que o atleta crie conceitos de "adaptação" a esse mundo de luta pelo resultado.

Face à área que abrange a prática desportiva de competição, Endler (1978), Davison e Schwartz (1976) citado por Martens, Vealey e Burton (1990) a ansiedade pode manifestar-se de duas formas: Ansiedade Cognitiva e Ansiedade Somática. Segundo Martens et al. (1990), a Ansiedade Cognitiva consiste em pensamentos negativos e preocupações sobre a performance, incapacidade para se concentrar e atenção disruptiva. Já a Ansiedade Somática é uma percepção das manifestações físicas da ativação, caracterizada por sensações físicas. A autoconfiança é conceituada por Machado (2006) como uma crença geral do indivíduo, que pode realizar com sucesso uma determinada ação, atividade.

Diante destes pressupostos, o objetivo deste estudo foi avaliar o grau de ansiedade pré-competitiva de atletas de natação de alto rendimento. Este estudo se mostra muito importante diante da escassez de dados desta natureza, especialmente nesta modalidade. 


\section{Metodologia}

O estudo foi realizado com uma população de atletas de natação do estado da Paraíba que treina com fins competitivos. A amostra foi constituída por 37 atletas, sendo 21 do sexo feminino e, com idades entre 13 e 23 anos, de três clubes da cidade de João Pessoa-PB.

\section{Instrumentos e Procedimentos na Recolha dos Dados}

O instrumento utilizado foi um questionário denominado Inventário do Estado de Ansiedade Competitiva - II (CSAI-2), traduzido e validado para português por Raposo (2004). O questionário é composto por 27 questões, são divididas em 3 subescalas (ansiedade cognitiva, ansiedade somática e auto-confiança), nas quais o sujeito opta por quatro graus correspondente ao seu estado momentâneo: 1 = nada, 2 =alguma coisa, 3 = moderado e 4 = muito, de acordo com a pergunta. A pontuação das subescalas é obtida pela somatória das respostas, com pontuação variando de 9 a 36. Para propósitos de interpretação, os dados da ansiedade cognitiva, somática e autoconfiança são categorizados em baixa (de 9 a 18 pontos), média de (19 a 27 pontos) e alta de (28 a 36 pontos).

A coleta de dados foi realizada em duas competições de nível distinto. Uma de nível apenas estadual Campeonato Paraibano de Clubes Infantil, Juvenil, Júnior I e Júnior II/Sênior- (CP), e outra de nível regional - XII Torneio Nordeste de Clubes Infantil, Juvenil, Júnior I e Júnior II/Sênior - Troféu Sérgio Silva $(\mathrm{CN})$.

Previamente à coleta dos dados, o pesquisador entrou em contato pessoal com os técnicos responsáveis pelos três clubes, obtendo deles permissão para realização do estudo. Foi solicitado ao técnico que avisasse a seus atletas e aos seus pais que eles seriam abordados no dia da competição, momentos antes de suas provas, para responder ao questionário.

O questionário foi respondido pelos próprios atletas com 15 minutos antes de suas provas. Para garantir a efetividade deste procedimento, o pesquisador se posicionou no banco de controle. Este é um local para onde os atletas de dirigem momentos antes da prova, de modo que este posicionamento garantiu que os dados fossem coletas efetivamente com 15 minutos antes do início das provas.

Embora alguns atletas participassem de mais de uma prova nestes eventos, eles responderam ao instrumento apenas na primeira vez em que foi abordado pelo pesquisador. 
Para responder o instrumento, o atleta tinha disponível cadeiras para se acomodar confortavelmente, e sempre foram informados que o tempo necessário para preencher o questionário era bastante curto. Na prática eles levaram entre cinco e dez minutos para esta tarefa. Quando eles chegavam ao banco de controle junto com pais, o pesquisador solicitava que os se afastassem momentaneamente, para evitar interferências nas respostas dos filhos.

Os dados foram tratados por meio de estatística descritiva (média e desvio padrão). Uma planilha foi construída por meio do software Excel XP.

\section{Resultados}

A Tabela 1, apresenta os dados de ansiedade e auto-confiança nas duas competições analisadas. Observase que os atletas demonstraram maiores níveis de ansiedade na competição de maior nível. No entanto a auto-confiança também foi maior. Outro aspecto importante que nas duas competições, o nível de autoconfiança foi bem maior que o nível de ansiedade.

Tabela 1- Média atingida pelos atletas nas duas competições estudadas. Os valores obtidos devem ser comparados com o critério de categorização (baixo: 9 a 18 pontos; médio: 19 a 27 pontos; alto: 28 a 36 pontos).

\begin{tabular}{lll}
\hline & $\begin{array}{l}\text { Campeonato } \\
\text { Paraibano (pontos) }\end{array}$ & $\begin{array}{l}\text { Campeonato } \\
\text { (pontos) }\end{array}$ \\
\hline $\begin{array}{l}\text { Ansiedade } \\
\text { cognitiva }\end{array}$ & $17(+-3,9)$ & $24,2(+-5,1)$ \\
Ansiedade & $16,2(+-3,5)$ & $20,5(+-4,5)$ \\
somática & & \\
Auto-confiança & $23,5(+-5,4)$ & $26,3(+-4,6)$ \\
\hline
\end{tabular}

A Tabela 2 mostra a distribuição dos atletas quanto a classificação dos níveis de ansiedade autoconfiança. Estes dados confirmam o fato de que competições de maior porte resultam em maior prevalecia de níveis ansiogênicos, mas que a auto-confiança é elevada independentemente do nível da competição. 
Tabela 2- Percentual de atletas com escores entre baixo, médio e alto para as três subescalas analisadas nas duas competições.

\begin{tabular}{lllllll}
\hline & \multicolumn{3}{l}{ Campeonato Paraibano (\%) } & \multicolumn{3}{c}{ Campeonato Regional (\%) } \\
\hline & Baixa & Média & Alta & Baixa & Média & Alta \\
Ansiedade Cognitiva & 80 & 20 & 0 & 17 & 53 & 30 \\
Ansiedade somática & 75 & 25 & 0 & 35 & 58 & 6 \\
Auto-confiança & 10 & 70 & 20 & 11 & 41 & 48 \\
\hline
\end{tabular}

Uma análise foi feita com os atletas sendo categorizados pelo gênero. Estes resultados estão expressos na tabelas 3, onde observa-se que homens e mulheres obtiveram pontuação muito similar para as três subescalas no CP. No entanto, na competição de mais alto nível, as homens obtiveram pontuação ligeiramente superiores aos homens para as três subescalas.

Tabela 3: comparação dos escores de ansiedade e auto-confiança entre homens e mulheres nas duas competições analisadas.

\begin{tabular}{lllll}
\hline & Campeonato Paraibano (pontos) & $\begin{array}{l}\text { Campeonato } \\
\text { (pontos) }\end{array}$ & Regional \\
& Fem & Masc & Fem & Masc \\
Ansiedade & $17,0(+-3,0)$ & $17,2(+-4,5)$ & $23,9(+-4,9)$ & $24,7(+-5,6)$ \\
$\begin{array}{l}\text { Cognitiva } \\
\text { Ansiedade }\end{array}$ & $15,8(+-2,4)$ & $16,5(+-4,2)$ & $19,2(+-4,0)$ & $22,3(+-4,8)$ \\
$\begin{array}{l}\text { somática } \\
\text { Auto-confiança }\end{array}$ & $24,7(+-4,3)$ & $22,5(+-6,0)$ & $24,8(+-5,5)$ & $28,4(+-1,1)$ \\
\hline
\end{tabular}

\section{Discussão}

Percebeu-se diferença entre os dois campeonatos ( $\mathrm{CP}$ e $\mathrm{CR}$ ), sendo um com traços de nível baixo de ansiedade (CP) e com nível mediano de ansiedade (CR). Isto deve-se principalmente ao grau de competitividade de cada evento. Para Freschknecht apud Freitas et al. (1999, p.10), o atleta tem receio, medo de falhar e, conseqüentemente, há um aumento na ansiedade. Stefanello apud Freitas et al. (1999) afirma que o ambiente do esporte competitivo se torna ameaçador para o indivíduo, visto que, o que está em jogo no momento, não é apenas uma vitória, mas sim importantes valores e metas próprias.

Percebemos também que a ansiedade cognitiva subiu de uma competição para outra devido ao medo, já 
que para Cruz apud Sanches et al., (2004) a ansiedade cognitiva caracteriza-se por expectativas negativas, perda de concentração e pensamentos de fracasso. A ansiedade somática relaciona-se coma percepção de sintomas fisiológicos como: tensão, taquicardia, mãos úmidas.

Os nadadores paraibanos possuem um bom nível de auto-confiança o que facilitará para estes durante futuras provas pois para Gusmán et al. apud Freitas et al. (1999) se o atleta possui um baixo nível de autoconfiança, o mesmo poderá desenvolver um maior número de falhas e, então, a ansiedade não diminuirá, pois a preocupação irá aumentar, causando uma menor concentração. Saber direcionar é a melhor maneira de administrar uma ansiedade pré-competitiva (OPPERMANN, 2004).

Para Martens et al. (1990), a autoconfiança, relaciona-se negativamente com a somática e a cognitiva, ou seja, se a cognitiva e a somática aumentavam a autoconfiança diminui, este fato não ocorreu com a amostra, possivelmente porque os atletas conseguem trabalhar a sua auto-confiança de acordo com o grau da competição. Para Perrault e Vallerand (1998), Gould et al. (1999), no que se refere à performance individual dos atletas a motivação e a confiança estão presentes no momento da competição, quando eles conseguem manter um alto nível de motivação tendem a ter uma boa performance.

A maior ansiedade encontrada entre os homens são um dado em contrário corrobora com a literatura com atletas de vôlei (STEFANELLO, 1990), no atletismo (DE ROSE JUNIOR; VASCONCELLOS, 1997), juntamente com os dados de Ucha et al. (2001), que pesquisou 11 modalidades, nas quais as mulheres apresentaram índices de ansiedade mais elevados que os homens.

Devido a escassez de estudos dessa natureza, faz-se necessária a realização de novos estudos que apresentem objetivos semelhantes. Elevando o numero de pesquisas envolvendo variáveis intervenientes no estado de ansiedade pré-competitiva em atletas de alto rendimento propiciando uma relevante contribuição para os próprios atletas e à comissão técnica.

\section{Conclusão}

Com o presente estudo pudemos verificaram que os atletas paraibanos de natação encontram-se num grau intermediário de ansiedade pré-competitiva podendo subir a medida que cresce o grau de importância das provas e diferente das informações encontradas na literatura, os atletas do sexo masculino tendem a ficar mais nervosos dos que as do sexo feminino em competições mais importante. 


\section{Referências}

CRUZ, J. Stress e ansiedade na competição desportiva: natureza, efeitos e avaliação. In: CRUZ, J. (Ed.). Manual de Psicologia do Desporto. Braga: SHO., 1996b. p. 148-214.

DAMÁZIO, W. A ansiedade no voleibol. 1997. Monografia (Trabalho de Conclusão de Curso) - Instituto de Biociências, Universidade Estadual Paulista, Rio Claro, 1997.

DE ROSE JUNIOR, D.; VASCONCELLOS, E. G. Ansiedade-traço competitiva e atletismo: um estudo com atletas infanto-juvenis. Revista Paulista de Educação Física, São Paulo, v. 11, n. 2, p. 148-154, jul./dez. 1997.

FREITAS, F. J. P. de. et al. A ansiedade pré-competitiva e o comportamento de autocontrole em jogadores de futsal. Revista do Curso de Psicologia, Canoas, n. 9, jan./jun. 1999. Disponível em: $<$ www.ulbra.br/psicologia/aletheia9.pdf $>$. Acesso em: 22 nov. 2006.

GONÇALVES, M. J. Estudo da ansiedade no rendimento desportivo, atendendo à ansiedade cognitiva, somática e auto-confiança nas modalidades de atletismo e basquetebol em ambos os sexos aos níveis da pré-competição e competição. 1998. Dissertação (Mestrado) - Faculdade de Ciências e Educação Física , Universidade do Porto, Porto 1998.

GOULD, D. at al. , Factors affecting olympic performance: Perceptions of athletes and coaches from more and less successful teams. The Sports Psychology, 13, 371-394, 1999.

HACKFORT, D.; SPIELBERGER, C. Sport-related anxiety: Current trends in theory and research. In:

. (Ed.). Anxiety in sports: on internacional perspective. New York: Hemisphere, 1989. p. 261267.

MACHADO, A. A. Psicologia do esporte: da educação física escolar ao treinamento esportivo. São Paulo: Guanabara Koogan, 2006.

MARTENS, R.; VEALEY, R.; BURTON, D. (Ed.). Competitive anxiety in sport. Champaign, In: Human Kinetics, 1990.

OPPERMANN, P. R. V. Medicina Esportiva. Psicologia: ansiedade pré-competitiva. São Paulo, outubro de 2004. Disponível em: <www.ativo.com/materias>. Acesso em: 22 nov. 2006.

PERREAULT, S.; VALLERAND, R. J. Coming from behind: on the effect of psychological moment on sport performance. Journal of Sport and Exercises Psycholgy, v. 20, p. 421-436.

RAPOSO, J. V.; FERNANDES, H. Análise confirmatória do CSAI-2. Lisboa: Ed da Univ de Tróis-osMontes e Alto Douro, (2004).

SCANLAN, T. K.; LEWTHWAITE, R. Social psychological aspects of competition for male youth sport participants: I. predictors of competitive stress. Journal of Sport Psychology, n. 6, p. 208-226. 
SANCHES, A. B.; CASAL, H. M. V.; BRANDÃO, M. R. F. Fatores de estresse no futebol. Revista Digital, Buenos Aires, ano 10, n. 73, jun. 2004.

STEFANELLO, J. M. F. Ansiedade competitiva e os fatores de personalidade de adolescentes que praticam voleibol: um estudo causal comparativo. Santa Maria. Kinesis, v. 6, n. 2, p. 203-24, 1990.

UCHA, F.G. et al. Ansiedad e indicadores de rendimiento em deportistas. Revista Digital, Buenos Aires, ano 6, n. 33, mar. 2001. 


\section{Marcella Manfrin Barbacena}

marcellamb@gmail.com

Fone: 0xx 8332448555 /Cel: 0xx 8388148556

\section{Universidade Federal da Paraíba}

\section{Roseni Nunes de Figueiredo Grisi}

rosenigrisi@hotmail.com

Fone: 0xx 8332167030 (DEF) \Cel: 0xx 8393323244

Universidade Federal da Paraíba 


\title{
NÍVEL DE ANSIEDADE PRÉ-COMPETITIVA EM ATLETAS DE NATAÇÃO
}

Marcella Manfrin Barbacena

Roseni Nunes de Figueiredo Grisi

\section{Resumo}

O estudo visou avaliar os níveis de ansiedade pré-competitiva de atletas de natação em duas competições de diferentes níveis: estadual (CP) e regional (CR). Participaram do estudo 37 atletas, sendo 21 do sexo feminino, de três clubes do estado da Paraíba. Eles responderam ao questionário de ansiedade précompetitiva (CSAI-2) 15 minutos antes de suas provas. O teste revelou níveis de ansiedade précompetitiva maiores em competições mais importantes (CR), com 36,8\% dos sujeitos apresentando ansiedade média e alta em CR e apenas $11,5 \%$ na CP. Além disso, as mulheres apresentaram uma tendência para serem mais ansiogênicas que os homens. Todos os atletas apresentaram um bom nível de autoconfiança independentemente do nível da competição, mas com tendência para aumento de acordo com o nível da competição. Este trabalho contribui para uma reflexão da ansiedade em situações competitivas, possibilitando assim, conhecer como variam os estados de ansiedade e de autoconfiança antes de uma competição, sabendo que a ansiedade poderá afetar positivamente ou negativamente no rendimento posterior dos atletas, devendo ser motivo de atenção aos técnicos, psicólogos e estudiosos da área.

\section{Palavras-Chave}

Ansiedade; Natação; Gênero.

\section{LEVEL OF ANXIETY PRE-COMPETITIVE IN ATHLETES IN SWIMMING}

Marcella Manfrin Barbacena

Roseni Nunes de Figueiredo Grisi

\begin{abstract}
This study aimed to investigate the pre-competitive anxiety levels of swimming athletes in two distinct competitive levels: State (CP) and Region (CR). Participated to study 37 athletes, 21 femme in to three differents clubs to Paraíba State. Their answered the i pre-competitive anxiety 15 min before their swimming test. The inventory revealed significant higher pre-competitive levels in the more important competitions (CR), with $36,8 \%$ the athletes to present anxiety like medium and higher, and just $11,5 \%$ in CP. After this, the femme presented a inclination for more anxiety than men. For other side, all athletics presented a good level for self-esteem independent the competitive level, but with tendency to raise in accordance with the level of the competition. This work contributes for a reflection on the action of the anxiety in competitive situations, making possible to know how varies the states of anxiety and selfconfidence before a competition, knowing that this will be able to affect positive or negative the results of the athletes, having to be attention for the choach, psychologists and of the area.
\end{abstract}

\section{Key-Words}

Anxiety; Swimming; Genders. 


\section{INTRODUÇÃO}

Sendo um contexto natural com situações de elevada exigência e competitividade, o desporto de rendimento é usado pelos psicólogos para a compreensão do comportamento humano, das emoções e dos processos de adaptação humana (HACKFORT; SPIELBERGER, 1989). O sucesso, as críticas sofridas e a oportunidade tão esperada pelo atleta do alto rendimento, são algumas das suas tensões vivenciadas e, desta forma, pode ser alguns fatores ansiogênicos, pois dependem da percepção e da interpretação que cada pessoa tem dos acontecimentos (DAMÁZIO, 1997).

Um dos campos mais abordados pela Psicologia do Desporto é a ansiedade e as suas relações com o rendimento. A experiência de stress e ansiedade na competição desportiva constitui um problema usual e preocupante para todos aqueles que, direta ou indiretamente, se encontram envolvidos no desporto (CRUZ, 1996b; SCANLAN, 1984).

De acordo com as pesquisas de Parfitt apud Gonçalves (1998) a ansiedade não tem o mesmo efeito em todas as pessoas e nem em todos os esportes. A ansiedade somática está mais ligada a esportes de exigências aeróbicas (lutas, corridas, natação, etc), enquanto a ansiedade cognitiva estará mais ligada a esportes competitivos que envolvem estratégias (xadrez, golf, sinuca, etc). Nos desportos individuais, como a natação, a iniciativa, determinação, persistência, coragem e motivação são determinantes nos resultados, fazendo com que o atleta crie conceitos de "adaptação" a esse mundo de luta pelo resultado.

Face à área que abrange a prática desportiva de competição, Endler (1978), Davison e Schwartz (1976) citado por Martens, Vealey e Burton (1990) a ansiedade pode manifestar-se de duas formas: Ansiedade Cognitiva e Ansiedade Somática. Segundo Martens et al. (1990), a Ansiedade Cognitiva consiste em pensamentos negativos e preocupações sobre a performance, incapacidade para se concentrar e atenção disruptiva. Já a Ansiedade Somática é uma percepção das manifestações físicas da ativação, caracterizada por sensações físicas. A autoconfiança é conceituada por Machado (2006) como uma crença geral do indivíduo, que pode realizar com sucesso uma determinada ação, atividade.

Diante destes pressupostos, o objetivo deste estudo foi avaliar o grau de ansiedade pré-competitiva de atletas de natação de alto rendimento. Este estudo se mostra muito importante diante da escassez de dados desta natureza, especialmente nesta modalidade. 


\section{METODOLOGIA}

O estudo foi realizado com uma população de atletas de natação do estado da Paraíba que treina com fins competitivos. A amostra foi constituída por 37 atletas, sendo 21 do sexo feminino e, com idades entre 13 e 23 anos, de três clubes da cidade de João Pessoa-PB.

\section{INSTRUMENTOS E PROCEDIMENTOS NA RECOLHA DOS DADOS}

O instrumento utilizado foi um questionário denominado Inventário do Estado de Ansiedade Competitiva - II (CSAI-2), traduzido e validado para português por Raposo (2004). O questionário é composto por 27 questões, são divididas em 3 subescalas (ansiedade cognitiva, ansiedade somática e auto-confiança), nas quais o sujeito opta por quatro graus correspondente ao seu estado momentâneo: 1 = nada, 2 =alguma coisa, 3 = moderado e 4 = muito, de acordo com a pergunta. A pontuação das subescalas é obtida pela somatória das respostas, com pontuação variando de 9 a 36. Para propósitos de interpretação, os dados da ansiedade cognitiva, somática e autoconfiança são categorizados em baixa (de 9 a 18 pontos), média de (19 a 27 pontos) e alta de (28 a 36 pontos).

A coleta de dados foi realizada em duas competições de nível distinto. Uma de nível apenas estadual Campeonato Paraibano de Clubes Infantil, Juvenil, Júnior I e Júnior II/Sênior- (CP), e outra de nível regional - XII Torneio Nordeste de Clubes Infantil, Juvenil, Júnior I e Júnior II/Sênior - Troféu Sérgio Silva (CN).

Previamente à coleta dos dados, o pesquisador entrou em contato pessoal com os técnicos responsáveis pelos três clubes, obtendo deles permissão para realização do estudo. Foi solicitado ao técnico que avisasse a seus atletas e aos seus pais que eles seriam abordados no dia da competição, momentos antes de suas provas, para responder ao questionário.

O questionário foi respondido pelos próprios atletas com 15 minutos antes de suas provas. Para garantir a efetividade deste procedimento, o pesquisador se posicionou no banco de controle. Este é um local para onde os atletas de dirigem momentos antes da prova, de modo que este posicionamento garantiu que os dados fossem coletas efetivamente com 15 minutos antes do início das provas.

Embora alguns atletas participassem de mais de uma prova nestes eventos, eles responderam ao instrumento apenas na primeira vez em que foi abordado pelo pesquisador.

Para responder o instrumento, o atleta tinha disponível cadeiras para se acomodar confortavelmente, e 
sempre foram informados que o tempo necessário para preencher o questionário era bastante curto. $\mathrm{Na}$ prática eles levaram entre cinco e dez minutos para esta tarefa. Quando eles chegavam ao banco de controle junto com pais, o pesquisador solicitava que os se afastassem momentaneamente, para evitar interferências nas respostas dos filhos.

Os dados foram tratados por meio de estatística descritiva (média e desvio padrão). Uma planilha foi construída por meio do software Excel XP.

\section{RESULTADOS}

A Tabela 1, apresenta os dados de ansiedade e auto-confiança nas duas competições analisadas. Observase que os atletas demonstraram maiores níveis de ansiedade na competição de maior nível. No entanto a auto-confiança também foi maior. Outro aspecto importante que nas duas competições, o nível de autoconfiança foi bem maior que o nível de ansiedade.

Tabela 1- Média atingida pelos atletas nas duas competições estudadas. Os valores obtidos devem ser comparados com o critério de categorização (baixo: 9 a 18 pontos; médio: 19 a 27 pontos; alto: 28 a 36 pontos).

\begin{tabular}{llll}
\hline & $\begin{array}{l}\text { Campeonato } \\
\text { Paraibano (pontos) }\end{array}$ & $\begin{array}{l}\text { Campeonato } \\
\text { (pontos) }\end{array}$ & Regional \\
Ansiedade & $17(+-3,9)$ & $24,2(+-5,1)$ \\
cognitiva & $16,2(+-3,5)$ & $20,5(+-4,5)$ \\
\hline $\begin{array}{l}\text { Ansiedade } \\
\text { somática } \\
\text { Auto-confiança }\end{array}$ & $23,5(+-5,4)$ & $26,3(+-4,6)$ \\
\hline
\end{tabular}

\footnotetext{
A Tabela 2 mostra a distribuição dos atletas quanto a classificação dos níveis de ansiedade autoconfiança. Estes dados confirmam o fato de que competições de maior porte resultam em maior prevalecia de níveis ansiogênicos, mas que a auto-confiança é elevada independentemente do nível da competição.
} 
Tabela 2- Percentual de atletas com escores entre baixo, médio e alto para as três subescalas analisadas nas duas competições.

\begin{tabular}{lllllll}
\hline & \multicolumn{3}{c}{ Campeonato Paraibano (\%) } & \multicolumn{3}{c}{ Campeonato Regional (\%) } \\
& Baixa & Média & Alta & Baixa & Média & Alta \\
Ansiedade Cognitiva & 80 & 20 & 0 & 17 & 53 & 30 \\
\hline Ansiedade somática & 75 & 25 & 0 & 35 & 58 & 6 \\
Auto-confiança & 10 & 70 & 20 & 11 & 41 & 48 \\
\hline
\end{tabular}

Uma análise foi feita com os atletas sendo categorizados pelo gênero. Estes resultados estão expressos na tabelas 3, onde observa-se que homens e mulheres obtiveram pontuação muito similar para as três subescalas no CP. No entanto, na competição de mais alto nível, as homens obtiveram pontuação ligeiramente superiores aos homens para as três subescalas.

Tabela 3: comparação dos escores de ansiedade e auto-confiança entre homens e mulheres nas duas competições analisadas.

\begin{tabular}{|c|c|c|c|c|}
\hline & \multicolumn{2}{|c|}{ Campeonato Paraibano (pontos) } & Campeonato & Regional \\
\hline & Fem & Masc & Fem & Masc \\
\hline $\begin{array}{l}\text { Ansiedade } \\
\text { Cognitiva }\end{array}$ & $17,0(+-3,0)$ & $17,2(+-4,5)$ & $23,9(+-4,9)$ & $24,7(+-5,6)$ \\
\hline $\begin{array}{l}\text { Ansiedade } \\
\text { somática }\end{array}$ & $15,8(+-2,4)$ & $16,5(+-4,2)$ & $19,2(+-4,0)$ & $22,3(+-4,8)$ \\
\hline Auto-confiança & $24,7(+-4,3)$ & $22,5(+-6,0)$ & $24,8(+-5,5)$ & $28,4(+-1,1)$ \\
\hline
\end{tabular}

\section{DISCUSSÃO}

Percebeu-se diferença entre os dois campeonatos (CP e CR), sendo um com traços de nível baixo de ansiedade (CP) e com nível mediano de ansiedade (CR). Isto deve-se principalmente ao grau de competitividade de cada evento. Para Freschknecht apud Freitas et al. (1999, p.10), o atleta tem receio, medo de falhar e, conseqüentemente, há um aumento na ansiedade. Stefanello apud Freitas et al. (1999) afirma que o ambiente do esporte competitivo se torna ameaçador para o indivíduo, visto que, o que está em jogo no momento, não é apenas uma vitória, mas sim importantes valores e metas próprias. 
Percebemos também que a ansiedade cognitiva subiu de uma competição para outra devido ao medo, já que para Cruz apud Sanches et al., (2004) a ansiedade cognitiva caracteriza-se por expectativas negativas, perda de concentração e pensamentos de fracasso. A ansiedade somática relaciona-se coma percepção de sintomas fisiológicos como: tensão, taquicardia, mãos úmidas.

Os nadadores paraibanos possuem um bom nível de auto-confiança o que facilitará para estes durante futuras provas pois para Gusmán et al. apud Freitas et al. (1999) se o atleta possui um baixo nível de autoconfiança, o mesmo poderá desenvolver um maior número de falhas e, então, a ansiedade não diminuirá, pois a preocupação irá aumentar, causando uma menor concentração. Saber direcionar é a melhor maneira de administrar uma ansiedade pré-competitiva (OPPERMANN, 2004).

Para Martens et al. (1990), a autoconfiança, relaciona-se negativamente com a somática e a cognitiva, ou seja, se a cognitiva e a somática aumentavam a autoconfiança diminui, este fato não ocorreu com a amostra, possivelmente porque os atletas conseguem trabalhar a sua auto-confiança de acordo com o grau da competição. Para Perrault e Vallerand (1998), Gould et al. (1999), no que se refere à performance individual dos atletas a motivação e a confiança estão presentes no momento da competição, quando eles conseguem manter um alto nível de motivação tendem a ter uma boa performance.

A maior ansiedade encontrada entre os homens são um dado em contrário corrobora com a literatura com atletas de vôlei (STEFANELLO, 1990), no atletismo (DE ROSE JUNIOR; VASCONCELLOS, 1997), juntamente com os dados de Ucha et al. (2001), que pesquisou 11 modalidades, nas quais as mulheres apresentaram índices de ansiedade mais elevados que os homens.

Devido a escassez de estudos dessa natureza, faz-se necessária a realização de novos estudos que apresentem objetivos semelhantes. Elevando o numero de pesquisas envolvendo variáveis intervenientes no estado de ansiedade pré-competitiva em atletas de alto rendimento propiciando uma relevante contribuição para os próprios atletas e à comissão técnica.

\section{CONCLUSÃO}

Com o presente estudo pudemos verificaram que os atletas paraibanos de natação encontram-se num grau intermediário de ansiedade pré-competitiva podendo subir a medida que cresce o grau de importância das provas e diferente das informações encontradas na literatura, os atletas do sexo masculino tendem a ficar mais nervosos dos que as do sexo feminino em competições mais importante. 


\section{REFERÊNCIAS}

CRUZ, J. Stress e ansiedade na competição desportiva: natureza, efeitos e avaliação. In: CRUZ, J. (Ed.). Manual de Psicologia do Desporto. Braga: SHO., 1996b. p. 148-214.

DAMÁZIO, W. A ansiedade no voleibol. 1997. Monografia (Trabalho de Conclusão de Curso) - Instituto de Biociências, Universidade Estadual Paulista, Rio Claro, 1997.

DE ROSE JUNIOR, D.; VASCONCELLOS, E. G. Ansiedade-traço competitiva e atletismo: um estudo com atletas infanto-juvenis. Revista Paulista de Educação Física, São Paulo, v. 11, n. 2, p. 148-154, jul./dez. 1997.

FREITAS, F. J. P. de. et al. A ansiedade pré-competitiva e o comportamento de autocontrole em jogadores de futsal. Revista do Curso de Psicologia, Canoas, n. 9, jan./jun. 1999. Disponível em: $<$ www.ulbra.br/psicologia/aletheia9.pdf>. Acesso em: 22 nov. 2006.

GONÇALVES, M. J. Estudo da ansiedade no rendimento desportivo, atendendo à ansiedade cognitiva, somática e auto-confiança nas modalidades de atletismo e basquetebol em ambos os sexos aos níveis da pré-competição e competição. 1998. Dissertação (Mestrado) - Faculdade de Ciências e Educação Física, Universidade do Porto, Porto 1998.

GOULD, D. at al. , Factors affecting olympic performance: Perceptions of athletes and coaches from more and less successful teams. The Sports Psychology, 13, 371-394, 1999.

HACKFORT, D.; SPIELBERGER, C. Sport-related anxiety: Current trends in theory and research. In: . (Ed.). Anxiety in sports: on internacional perspective. New York: Hemisphere, 1989. p. 261267.

MACHADO, A. A. Psicologia do esporte: da educação física escolar ao treinamento esportivo. São Paulo: Guanabara Koogan, 2006.

MARTENS, R.; VEALEY, R.; BURTON, D. (Ed.). Competitive anxiety in sport. Champaign, In: Human Kinetics, 1990.

OPPERMANN, P. R. V. Medicina Esportiva. Psicologia: ansiedade pré-competitiva. São Paulo, outubro de 2004. Disponível em: <www.ativo.com/materias>. Acesso em: 22 nov. 2006.

PERREAULT, S.; VALLERAND, R. J. Coming from behind: on the effect of psychological moment on sport performance. Journal of Sport and Exercises Psycholgy, v. 20, p. 421-436.

RAPOSO, J. V.; FERNANDES, H. Análise confirmatória do CSAI-2. Lisboa: Ed da Univ de Tróis-osMontes e Alto Douro, (2004).

SCANLAN, T. K.; LEWTHWAITE, R. Social psychological aspects of competition for male youth sport 
participants: I. predictors of competitive stress. Journal of Sport Psychology, n. 6, p. 208-226.

SANCHES, A. B.; CASAL, H. M. V.; BRANDÃO, M. R. F. Fatores de estresse no futebol. Revista Digital, Buenos Aires, ano 10, n. 73, jun. 2004.

STEFANELLO, J. M. F. Ansiedade competitiva e os fatores de personalidade de adolescentes que praticam voleibol: um estudo causal comparativo. Santa Maria. Kinesis, v. 6, n. 2, p. 203-24, 1990.

UCHA, F.G. et al. Ansiedade e indicadores de rendimiento em deportistas. Revista Digital, Buenos Aires, ano 6, n. 33, mar. 2001. 


\section{Marcella Manfrin Barbacena}

Universidade Federal da Paraíba

\section{Roseni Nunes de Figueiredo Grisi}

Universidade Federal da Paraíba

\section{Referência do artigo:}

\section{ABNT}

BARBACENA, M. M., GRISI, R. N. F. Nível de ansiedade pré-competitiva em atletas de natação. Conexões, v. 6, n. 1, p. 31-39, 2008.

\section{APA}

Barbacena, M. M., \& Grisi, R. N. F. (2008). Nível de ansiedade pré-competitiva em atletas de natação. Conexões, 6(1), 31-39.

\section{VANCOUVER}

Barbacena MM, Grisi RNF. Nível de ansiedade pré-competitiva em atletas de natação. Conexões, 2008, 6(1): 31-39. 